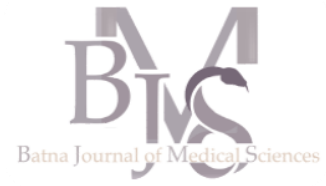

Service d'Endocrinologie et des maladies métaboliques, $\mathrm{CHU}$ Bab El Oued, Alger - Algérie.

\section{Correspondance à :}

Saïd AZZOUG

saidazzoug@yahoo.fr

DOI :https://doi.org/10.48087/ BJMSra.2016.3204

Il s'agit d'un article en libre accès distribué selon les termes de la licence Creative Commons Attribution International License (CC BY 4.0), qui autorise une utilisation, une distribution et une reproduction sans restriction sur tout support ou format, à condition que l'auteur original et la revue soient dûment crédités.

\title{
La crise aigue thyrotoxique
}

\section{Thyroid storm}

\section{Saïd Azzoug, Farida Chentli}

\section{RÉSUMÉ}

La crise aigue thyrotoxique est une complication rare mais grave de l'hyperthyroïdie. Elle est secondaire à la décompensation d'une hyperthyroïdie sous-jacente méconnue ou négligée. Les infections et le traitement chirurgical ou par l'iode radioactif sans préparation médicale préalable sont les facteurs déclenchants habituels. Elle entraine une défaillance multiviscérale où prédominent les signes généraux et cardiovasculaires. Son diagnostic est clinique, il repose sur la capacité de jugement du médecin traitant. Son traitement repose sur l'inhibition de la libération et de l'effet des hormones thyroïdiennes et le traitement des complications associées. La prévention par le diagnostic précoce et la prise en charge adéquate de toute hyperthyroïdie demeure le meilleur moyen pour améliorer le pronostic de cette affection.

Mots clés : crise aigue thyrotoxique ; hyperthyroïdie défaillance multiviscérale ; prévention

\begin{abstract}
Thyroid storm is a rare but threatening complication of hyperthyroidism. It is secondary to a decompensated underlying unknown or neglected hyperthyroidism. Infections and surgery or radioiodine therapy without medical preparation are the usual precipitating factors. It leads to multi organ failure where cardiovascular and systemic signs are the main symptoms. Its diagnosis is clinical; it depends on the clinical judgment of the treating physician. Its treatment consists on the inhibition of liberation and effects of thyroid hormones and treatment of associated complications. Prevention by a prompt diagnosis and adequate management of any hyperthyroidism is the best way to improve the prognosis of this affection.
\end{abstract}

Keywords: thyroid storm ; hyperthyroidism ; multi organ failure ; prevention

\section{نوبة حادة لتسمم الارقية}

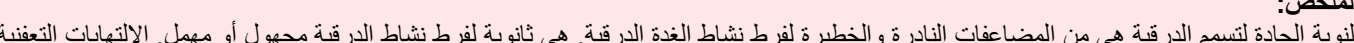

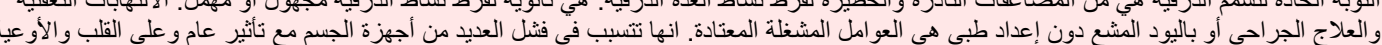

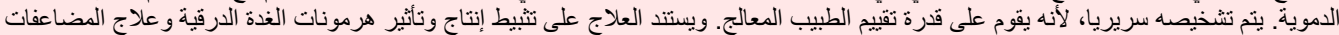

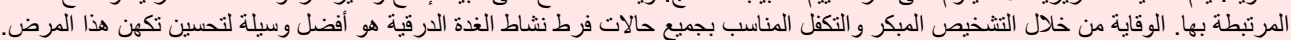
الكلمات المفتاحية: الحاد التسمم الدرقي الأزمة، فرط نشاط الغدة الدرقية، وفثل العديد من أجهزة الجسم والوقاية

\section{DÉFINITION}

La crise aigue thyrotoxique (CAT) est une complication rare mais grave de l'hyperthyroïdie, c'est l'accentuation extrême du tableau d'hyperthyroïdie.

\section{PATHOGÉNIE}

La pathogénie de la CAT demeure discutée, mais deux hypothèses sont proposées: une augmentation massive et brutale de la concentration des hormones thyroïdiennes libres [1], et une augmentation de la densité tissulaire des récepteurs béta adrénergiques ou une modification de la signalisation cellulaire post récepteur entrainant une hypersensibilité aux effets catécholaminergiques des hormones thyroïdiennes [2].

\section{ÉTIOLOGIES}

La CAT complique une hyperthyroïdie préexistante insuffisamment ou non traitée. Elle peut s'observer quelle que soit l'étiologie de l'hyperthyroïdie [3]. Habituellement, la CAT survient suite à un facteur déclenchant qui exacerbe les signes d'hyperthyroïdie.

Les facteurs déclenchants les plus incriminés sont [4] :

- Arrêt intempestif du traitement par antithyroïdiens de synthèse.

- Préparation médicale insuffisante à une chirurgie thyroïdienne (la CAT survient quelques heures après la thyroïdectomie) ou à un traitement par l'iode radioactif (dans ce cas la CAT survient quelques jours après l'administration de l'iode radioactif). Ceci 
Inhibition de la libération des hormones thyroïdiennes : administration de fortes doses d'iodure. L'iode doit être administré après avoir débuté les ATS (afin d'inhiber la synthèse d'hormones thyroïdiennes qui est initialement stimulé par l'iode), le délai entre les deux prises est discuté mais l'iode doit être débuté au moins 30 à 60 minutes après le début des ATS. L'iode inhibe la libération des hormones thyroïdiennes pré synthétisées, il inhibe également le captage et l'organification de l'iode. Cet effet connu sous le terme d'effet "Wolff-Chaikoff" dure 1 à 4 semaines, la thyroïde échappera secondairement à cet effet inhibiteur. Il est administré sous forme de solution de Lugol fort à $10 \%, 30$ à 100 gouttes per os toutes les 8 heures, ou sous forme de solution d'iodure de potassium, 5 gouttes per os toutes les 6 heures.

Inhibition des effets périphériques des hormones thyroïdiennes: Les bétabloquants sont un traitement essentiel de l'hyperthyroïdie par inhibition des effets périphériques catécholaminergiques notamment les effets cardiovasculaires des hormones des hormones thyroïdiennes. Le propranolol est le médicament de choix car en plus de son activité bétabloquante il diminue la conversion périphérique de T4 en T3 lorsqu'il est administré à fortes doses. Le propranolol est utilisé par voie orale à la dose de 20 à $80 \mathrm{mg}$ toutes les 4 à 6 heures, il peut également être administré par voie intraveineuse à la seringue électrique sous monitoring cardiaque à la dose de $0.5 \mathrm{mg}$ par heure après une dose de charge de $1 \mathrm{mg}$ en une minute. D'autres bétabloquants peuvent être utilisés comme l'atenolol à la dose de 50 à 200 $\mathrm{mg} /$ jour en une à deux prises ou le metoprolol à la dose de 100 à $200 \mathrm{mg} /$ jour en une à deux prises. L'esmolol, un bétabloquant d'action rapide peut aussi être utilisé dans cette situation.

Inhibition de la conversion périphérique de T4 en T3: Les glucocorticoïdes diminuent la conversion périphérique de T4 en T3. On utilise l'hydrocortisone $100 \mathrm{mg}$ en IV toutes les 6 à 8 heures ou la dexaméthasone $2 \mathrm{mg}$ en IV toutes les 6 heures. Ils peuvent également être utilisés par voie orale, sous forme de prednisone 50 à $100 \mathrm{mg}$ par jour ou de dexaméthasone $8 \mathrm{mg}$ par jour.

Thérapeutiques alternatives : Ces traitements peuvent être considérés en cas d'inefficacité, de non tolérance ou de contre indication des traitements précédents [10].

Lithium : Il inhibe la libération des hormones thyroïdiennes. Il est utilisé à la dose de $300 \mathrm{mg}$ per os toutes les 8 heures. Les concentrations plasmatiques de lithium (lithémie) doivent être maintenues entre 0.6 et $1 \mathrm{mEq} / \mathrm{l}$.

Perchlorate de potassium : Le perchlorate de potassium est un inhibiteur compétitif du transport d'iode, il inhibe la captation thyroïdienne de l'iode et favorise la libération de l'iode. Il est utilisé à la dose de $250 \mathrm{mg}$ per os 4 fois par jour. Son utilisation est limitée par le risque d'intoxication hématologique (anémie aplasique) et néphrologique (syndrome néphrotique), ce risque est cependant faible en cas d'utilisation de courte durée (moins de 4 semaines).

Cholestyramine: La cholestyramine est une résine échangeuse d'ions qui diminue la réabsorption des hormones thyroïdiennes dans la circulation entérohépatique, en effet, dans l'hyperthyroïdie la circulation entérohépatique des hormones thyroïdiennes est augmentée. Elle est utilisée à la dose de 4 gr per os toutes les 6 heures. Elle ne doit pas être administrée en même temps que les autres traitements pour ne pas diminuer leur absorption.
Plasmaphérèse: Les échanges plasmatiques par plasmaphérèse permettent une soustraction des hormones thyroïdiennes liées aux protéines de transport, ils permettent également de soustraire les anticorps anti-récepteur de la TSH diminuant ainsi l'intensité de l'hyperthyroïdie. C'est le seul traitement efficace en cas d'intoxication massive par les hormones thyroïdiennes. Son effet est partiel et transitoire, il dure 24 à 48 heures, d'où la nécessité de répéter les séances et d'envisager ce traitement en préparation à un traitement radical chirurgical.

\section{Mesures générales}

Fièvre: L'hyperthermie est fréquente dans la CAT, les moyens antipyrétiques physiques telle qu'une couverture réfrigérante est préconisée. Le paracétamol peut être utilisé, par contre les salicylés sont déconseillés car ils augmentent la fraction libre des hormones thyroïdiennes.

Réhydratation: Réhydratation par du sérum salé isotonique et du sérum glucosé avec apport en vitamine B1 (thiamine).

Traitement $d u$ facteur déclenchant: La recherche et le traitement d'un facteur déclenchant est essentiel dans la prise en charge de la CAT.

Déclaration d'intérêts: les auteurs ne déclarent aucun conflit d'intérêt en rapport avec cet article.

\section{RÉFÉRENCES}

1. Brooks $\mathrm{MH}$, Waldstein SS. Free thyroxine concentrations in thyroid storm. Ann Intern Med. 1980;93(5):694-7

2. Silva JE, Landsberg L. Catecholamines and the sympathoadrenal system in thyrotoxicosis. In: Braverman LE, Utiger RD, editors. Werner's \& Ingbar's The thyroid. 6th ed. Philadelphia: Lipincott, Williams \& Wilkins; 1991. p. 816-27

3. Bahn R, Burch H, Cooper D, et al. Hyperthyroidism and Other Causes of Thyrotoxicosis: Management Guidelines of the American Thyroid Association and American Association of Clinical Endocrinologists. Thyroid 2011; 21(6): 593-646

4. Nayak B, Burman K. Thyrotoxicosis and thyroid storm. Endocrinol Metab Clin North Am. 2006;35:663-86

5. Pimental L, Hansen K. Thyroid disease in the emergency department: a clinical and laboratory review. J Emerg Med. 2005;28:201-9

6. Dabon-Almirante $\mathrm{CL}$, Surks $\mathrm{M}$. Clinical and laboratory diagnosis of thyrotoxicosis. Endocrinol Metab Clin North Am. 1998;27(1):25-35

7. Burch HB, Wartofsky L. Life-threatening thyrotoxicosis. Thyroid storm. Endocrinol Metab Clin North Am 1993;22:263-77

8. Cooper DS. Antithyroid drugs. N Engl J Med. 2005;352:905-17

9. Cooper D. Treatment of thyrotoxicosis. In: Braverman LE, Utiger RD, editors. Werner's \& Ingbar's The thyroid. 9th ed. Philadelphia: Lipincott, Williams \& Wilkins; 2005. p. 665-94

10. Barbesino G. Drugs affecting thyroid function. Thyroid 2010;20(7):763-70 